\title{
Capacidad jurídica de las personas con discapacidad en el código civil y comercial
}

\author{
Por Marina M. Sorgi Rosenthal ${ }^{1}$
}

SUMARIO: I. Introducción. II. Concepto. III. Regulación en el Código Civil y Comercial. III. a) Restricciones de la capacidad. III. b) Proceso de determinación de incapacidad. III. c) Sistemas de apoyo al ejercicio de la capacidad. IV. Conclusión. V. Bibliografía.

Resumen: El presente artículo tiene como objetivo analizar el régimen de capacidad jurídica de las personas con discapacidad en la regulación del Código Civil y Comercial de la Nación, a la luz de las prerrogativas consagradas en la Convención sobre los Derechos de las Personas con Discapacidad. Para tal fin, expliqué sucintamente en que concierne el paradigma de modelo social de discapacidad receptado en el instrumento internacional mencionado anteriormente. Luego analicé las normas del Código unificado que regulan las restricciones a la capacidad, el proceso de determinación de la misma y los sistemas de apoyo. Para finalmente concluir, que el Código se ha adecuado a los estándares previstos en la Convención sobre los Derechos de las Personas con Discapacidad. Cabe señalar, que la metodología utilizada en este trabajo fue de tipo cualitativo a través de una revisión bibliográfica del área temática específica.

Palabras clave: Código Civil y Comercial; Discapacidad, Capacidad; Apoyos.

Abstract: The present article aims to analyze the legal capacity system of persons with disabilities in the regulation of the Civil and Commercial Code of the Nation, in light

\footnotetext{
${ }^{1}$ Abogada y escribana egresada de la Facultad de Ciencias Jurídicas y Sociales de la Universidad Nacional de La Plata. Maestreando en Derechos Humanos de dicha Universidad (en proceso de titulación). Doctorando en Cs. Sociales (FaHCE). Becaria Tipo A de la Universidad Nacional de La Plata. Grupo de Estudio de la Complejidad en la Sociedad de la Información. Dirección postal de correspondencia: 467 No 425 entre 14 y 14 A. E-mail: mar sorgi@yahoo.com.ar Teléfonos de contacto: (0221) $156806607 /(0221) 480-2015$
} 
of the prerogatives enshrined in the Convention on the Rights of Persons with Disabilities. For that purpose I briefly explained the social model paradigm of disability received in the international instrument mentioned above. Then I analyze the rules of the Unified Code that regulate the capacity constraints, the process of determining it and the support systems. To finally conclude that the Code has adapted to the standards set forth in the Convention on the Rights of Persons with Disabilities. It should be noted that the methodology used in this work was qualitative through a bibliographic review of the specific thematic area.

Keywords: Civil and Commercial Code; Disability; Capacity ; Support

\section{Introducción}

La República Argentina receptó el Modelo Social de discapacidad a partir de la adopción de la Convención sobre los Derechos de las Personas con Discapacidad, mediante la Ley $\mathrm{N}^{\circ} 26.378$, la cual tiene jerarquía constitucional a través de la Ley $\mathrm{N}^{\circ}$ 27.044 .

Este nuevo paradigma sostiene que la noción de persona con discapacidad se basa, más allá de la diversidad funcional de las personas, en las limitaciones de la propia sociedad. Razón por la que, las soluciones dadas no deben apuntarse individualmente a la persona afectada, sino más bien se encuentran dirigidas hacia la sociedad, o teniendo muy presente el contexto social en el cual la persona desarrolla su vida, erigiéndose como herramientas fundamentales el diseño universal y la accesibilidad.

Por su parte, la Convención (2006) consagra en su artículo 12 el derecho al Igual reconocimiento como persona ante la ley, el cual comprende el reconocimiento de su personalidad jurídica (inc. 1), el ejercicio de la capacidad jurídica en igualdad de condiciones con las demás en todos los aspectos de la vida (inc. 2), la adopción de medidas para proporcionar el acceso de apoyos (inc. 3), la adopción de salvaguardias para asegurar el ejercicio de la capacidad jurídica, las cuales deben respetar los derechos, la voluntad y las preferencias de la persona, que no haya conflicto de intereses ni influencia indebida, que sean proporcionales y adaptadas a las circunstancias de la persona, que se apliquen en el plazo más corto posible y que estén sujetas a exámenes periódicos por parte de una autoridad o un órgano judicial competente, independiente e imparcial (inc. 4).

También tiene como objetivo garantizar el derecho a ser propietarias, controlar sus asuntos económicos y tener acceso a crédito financiero (inc. 5).

Además, prevé entre sus principios generales, la no discriminación, el respeto de la dignidad inherente, la autonomía individual, incluida la libertad de tomar las propias decisiones, y la independencia de las personas (art. 3). 
El Comité sobre los Derechos de las Personas con Discapacidad [CRPD] (2014), como órgano encargado de interpretar la Convención, ha señalado que:

Los Estados deben adoptar las medidas siguientes para asegurar la plena aplicación del artículo 12 de la Convención sobre los derechos de las personas con discapacidad:

a) Reconocer a las personas con discapacidad como personas ante la ley, con personalidad jurídica y capacidad jurídica en todos los aspectos de la vida, en igualdad de condiciones con las demás. Ello exige suprimir los regímenes y mecanismos basados en la sustitución en la adopción de decisiones que niegan la capacidad jurídica con el propósito o el efecto de discriminar a las personas con discapacidad.

b) Establecer, reconocer y proporcionar a las personas con discapacidad el acceso a una amplia gama de apoyos en el ejercicio de su capacidad jurídica. Las salvaguardias para esos apoyos deben estar fundadas en el respeto de los derechos, la voluntad y las preferencias de las personas con discapacidad. (p.47)

Por lo que el presente trabajo tiene como objetivo analizar el régimen de la capacidad jurídica en el Código Civil y Comercial de la Nación a la luz de los estándares y obligaciones asumidas por nuestro Estado en materia de derechos de las personas con discapacidad.

\section{Concepto}

Etimológicamente la palabra capacidad viene del latín capacitatis, característica esta que posee el capax o capaz el cual tiene como derivativo el verbo capio que significa tomar, agarrar, apoderarse. El capaz en esta lógica sería aquella persona que tiene la posibilidad de tomar o asumir actividades acordes a su habilidad o destreza. En el derecho romano el caput (literalmente cabeza) hacía referencia al estatus de las personas físicas, cuya posición determinaba la capacidad jurídica, siendo la capitis deminutio la disminución de la capacidad, que llevada a su grado máximo conducía a la pérdida de la libertad (Mackeldey, 1844).

De acuerdo con el viejo Código Civil de Vélez Sarsfield, Guillermo A. Borda (1999) definía a la capacidad como "la aptitud de las personas para adquirir derechos y contraer obligaciones”.(p. 454) Y a continuación añadía que:

Es fácil advertir la estrechísima relación que existe entre estado y capacidad, puesto que el estado es la base sobre la que descansa la capacidad: de aquél dependen los derechos y obligaciones de una persona. El estado es, pues, la estática y la capacidad, la dinámica de un mismo problema: los derechos y deberes jurídicos de las personas. La capacidad puede referirse al goce de los derechos o a su ejercicio; en el primer caso, se trata de capacidad de derecho; en el segundo, de capacidad de hecho. (p. 454) 
En tanto que Santos Cifuentes (1992) la referenciaba como "la aptitud o posibilidad jurídica de gozar y obrar los derechos. Se divide en capacidad de derecho, aptitud de ser titular, y capacidad de hecho, aptitud para ejercer los derechos por sí mismo" (p.169).

En armonía con el nuevo Código podríamos decir que la capacidad es la regla general por la cual toda persona humana goza de la posibilidad jurídica de ser titular de derechos y deberes jurídicos sólo pudiendo ser limitada o privada por ley respecto a determinados hechos, simples actos o actos jurídicos y a ejercerlos por sí mismo con las limitaciones expresamente previstas en el Código y en una sentencia judicial.

\section{Regulación de la capacidad en el Código Civil y Comercial}

El Código Civil y Comercial (2014) regula la capacidad en el libro primero, título I, capítulo 2 y dividiendo al mismo en tres secciones: principios generales, persona menores de edad y restricciones a la capacidad.

La comisión redactora del Código, integrada por Lorenzetti, R.; Higton de Nolasco, E. \& Kemelmajer de Carlucci, A (2012), en sus fundamentos señala que:

El Anteproyecto distingue la capacidad de derecho de la capacidad de ejercicio. Las modificaciones importantes se producen en esta última, a fin de adecuar el derecho positivo a la Convención Internacional de los derechos del niño y a la Convención Internacional de las personas con discapacidad. (p.544).

En efecto, en su art. 22 el Código dice que "toda persona humana goza de la aptitud para ser titular de derechos y deberes jurídicos", por lo que la capacidad es la regla siendo los casos establecidos en la ley, sus excepciones. En este sentido, "la ley puede privar o limitar esta capacidad respecto de hechos, simples actos, o actos jurídicos determinados" (Lorenzetti, R. et al., 2012: 544).

La capacidad de ejercicio regulada en el artículo 23 sigue la misma regla del ejercicio por parte de toda persona humana pero reduciendo las limitaciones a las expresamente contempladas en el propio Código y en una sentencia judicial. Estas son: la persona por nacer, la persona que no cuenta con la edad y grado de madurez suficiente y, la persona declarada incapaz por sentencia judicial, en la extensión dispuesta en esa decisión.

Según los codificadores (Lorenzetti, R. et al., 2012), la adecuación de este cuerpo normativo a las Convenciones Internacionales en este punto explica que:

La flexibilidad de las normas, las permanentes referencias a nociones como 'edad y grado de madurez', la necesidad de que las restricciones a la capacidad estén legalmente previstas, las facultades judiciales para la determinación de esas 
restricciones, la obligación del juez de oír, tener en cuenta y valorar las opiniones de estas personas, etc. (544).

\section{II. a) Restricciones a la capacidad}

El Código Civil y Comercial de la Nación (2014) regula en su artículo 31 las reglas generales de la restricción al ejercicio de la capacidad jurídica. Presume la capacidad general de ejercicio de la persona humana y que las limitaciones a la capacidad son de carácter excepcional y se imponen siempre en beneficio de la persona. Cabe aclarar al respecto que "esta regla-límite del "beneficio de la persona" debe verse sometida a un estricto test de proporcionalidad, que descarte los eventuales abusos en el marco de un paternalismo estatal injustificado cuyo resultado es la violación de los derechos de las personas" (Fernández: 2015).

El artículo citado, además, expresa que la intervención estatal deberá tener siempre carácter interdisciplinario, siendo un derecho de la persona con discapacidad recibir información de forma comprensible.

Asimismo, establece que la persona tiene derecho a participar en el proceso judicial con asistencia letrada y que deben priorizarse las alternativas terapéuticas menos restrictivas de sus derechos y libertades.

Por otro lado, en el artículo 32, prevé que el juez puede restringir la capacidad para determinados actos de una persona mayor de trece años que padece una adicción o una alteración mental permanente o prolongada, de suficiente gravedad, siempre que estime que del ejercicio de su plena capacidad puede resultar un daño a su persona o a sus bienes, debiendo designar el o los apoyos necesarios en relación a dichos actos. Estableciendo que sólo excepcionalmente, en el supuesto de que la persona se encuentre absolutamente imposibilitada de interaccionar con su entorno y de expresar su voluntad por cualquier modo y el sistema de apoyos resulte ineficaz, el juez puede declarar la incapacidad y designar un curador.

Sin embargo, cabe resaltar que este último supuesto sólo podrá darse excepcionalmente, ya que "el hecho de que una persona tenga una discapacidad o una deficiencia (incluidas las deficiencias físicas o sensoriales) no debe ser nunca motivo para negarle la capacidad jurídica ni ninguno de los derechos establecidos en el artículo 12" (CRDP, 2014: 9).

\section{II. b) Proceso de determinación de incapacidad}

En cuanto a la legitimación activa, el Código prevé que pueden solicitar la declaración de incapacidad y de capacidad restringida (art. 33): 
a) el propio interesado

b) el cónyuge no separado de hecho y el conviviente mientras la convivencia no haya cesado

c) los parientes dentro del cuarto grado; si fueran por afinidad, dentro del segundo grado y

d) el Ministerio Público.

Ornela Piccinelli (2015) comenta que:

No cabe duda de que el nuevo modelo, conteste con la regla de la presunción de la capacidad que propone, explicita que la persona en beneficio de quien se promueve el proceso reviste la calidad de parte. De allí que se disponga que puede aportar todas las pruebas que hacen a su defensa y que si la persona ha comparecido sin abogado, se le debe nombrar uno para que la represente y le preste asistencia letrada en el juicio. Aparece ratificada la figura del abogado del art. 22 de la Ley de Salud Mental, que deberá cohonestarse con las previsiones del Código Procesal Civil y Comercial en punto a la utilización de la tradicional figura del curador provisional (s.p).

El artículo 22 de la Ley N²6657 (2010) dispone que:

La persona internada involuntariamente o su representante legal, tiene derecho a designar un abogado. Si no lo hiciera, el Estado debe proporcionarle uno desde el momento de la internación. El defensor podrá oponerse a la internación y solicitar la externación en cualquier momento. El juzgado deberá permitir al defensor el control de las actuaciones en todo momento.

Esto requiere la inmediación judicial efectiva donde las partes tengan contacto directo con el juez, sin intermediarios y la oralidad viene a completar este marco donde se analicen de primera mano las pruebas aportadas y los intereses de las partes intervinientes.

Es por ello que durante el transcurso del proceso, el juez debe ordenar las medidas cautelares necesarias para garantizar los derechos personales y patrimoniales de la persona, debiendo determinar qué actos requieren la asistencia de apoyos, y cuáles la representación de un curador.

Asimismo, como medida de salvaguarda, y acorde al principio de autonomía de las personas con discapacidad, el código prevé que la misma sea parte del proceso (Art. 36) y que el juez deberá garantizar la inmediatez y entrevistarla personalmente, asegurando la accesibilidad y los ajustes razonables del procedimiento de acuerdo a la 
situación de aquella (art. 35). Esto último se condice con lo previsto en el artículo 13 de la Convención sobre los Derechos de las Personas con Discapacidad que consagra el derecho de acceso a la justicia. Asimismo, debe ser complementado con lo normado por el artículo 707 del Código Civil y Comercial que expresa que:

Las personas mayores con capacidad restringida y los niños, niñas y adolescentes tienen derecho a ser oídos en todos los procesos que los afectan directamente. Su opinión debe ser tenida en cuenta y valorada según su grado de discernimiento y la cuestión debatida en el proceso.

A su vez, establece en el artículo 37 los aspectos que debe contemplar la sentencia de restricción de la capacidad, entre los que se encuentra el diagnóstico y pronóstico; la época en que la situación se manifestó; los recursos personales, familiares y sociales existentes y el régimen para la protección, asistencia y promoción de la mayor autonomía posible, siendo imprescindible para expedirse el dictamen de un equipo interdisciplinario.

Este artículo debe interpretarse a la luz de la Convención sobre los Derechos de las Personas con Discapacidad que recepta el modelo social de discapacidad. Por lo que el diagnóstico, que es propio del modelo médicorehabilitador, no deberá ser el elemento determinante a la hora de restringir la capacidad de las personas.

Recordemos, que tal como ha afirmado el Comité sobre los Derechos de las Personas con Discapacidad, no deben mezclarse los conceptos de capacidad mental y capacidad jurídica. Esto debido a que:

La capacidad mental se refiere a la aptitud de una persona para adoptar decisiones, que naturalmente varía de una persona a otra y puede ser diferente para una persona determinada en función de muchos factores, entre ellos factores ambientales y sociales (CRDP, 2014: 12).

Por lo que, de conformidad con el artículo 12 de la Convención, los déficits en la capacidad mental no deben utilizarse como justificación para negar la capacidad jurídica (CRDP, 2014: 12).

Por otro lado, la sentencia deberá determinar la extensión y alcance de la restricción y especificar las funciones y actos que se limitan, procurando que la afectación de la autonomía personal sea la menor posible. Asimismo, debe designar una o más personas de apoyo o curadores y señalar las condiciones de validez de los actos específicos sujetos a la restricción con indicación de la o las personas intervinientes y la modalidad de su actuación (art. 38). Así como también, ser inscripta en el Registro de Estado Civil y Capacidad de las Personas (art. 39).

La resolución judicial podrá ser revisada en cualquier momento a instancias del interesado (art. 40). Previendo a su vez, que en los casos normados por el artículo 32 
la sentencia debe ser revisada por el juez en un plazo no superior a tres años, sobre la base de nuevos dictámenes interdisciplinarios y mediando la audiencia personal con el interesado. Poniendo en cabeza del Ministerio Público la obligación de fiscalizar el cumplimiento efectivo de la revisión judicial y en caso contrario instarla a que ésta se lleve a cabo. De lo que se desprende, que este artículo concuerda con lo dispuesto en el artículo 7 inciso $\mathrm{n}$ de la Ley $\mathrm{N}^{\circ} 26.657$ que afirma que el Estado reconoce el derecho a que el padecimiento mental no sea considerado un estado inmodificable.

En este orden de ideas, cabe señalar que en el proceso de revisión de sentencia deberán respetarse todas las garantías procesales. Es decir, que:

La persona tiene derecho a conocer que será evaluada y a qué fines, el resultado de la pericia (de la cual deben correrle los traslados correspondientes), impugnarla, presentar las pruebas que se consideren pertinentes, solicitar la celebración y una audiencia con el juez, ofrecer consultores técnicos, entre otros. En definitiva, debe primar una interpretación que procure la mayor amplitud posible de las garantías procesales. (Ferraiuolo, A \& Crocci, S: 2015).

Cabe destacar que el Código limita la internación sin consentimiento de la persona a que se cumplan los recaudos previstos en el mismo (art. 41):

a) Evaluación de un equipo interdisciplinario que señale los motivos que la justifican y la ausencia de una alternativa eficaz menos restrictiva de su libertad;

b) La existencia de riesgo cierto e inminente de un daño de entidad para la persona protegida o para terceros;

c) Es un recurso terapéutico de carácter restrictivo y debe ser supervisada periódicamente y por el menor tiempo posible

d) Debe garantizarse el debido proceso, el control judicial inmediato y el derecho de defensa mediante asistencia jurídica; y

e) La sentencia que aprueba la internación debe especificar su finalidad, duración y periodicidad de la revisión.

La Corte Suprema de Justicia de la Nación (2008) ha dicho al respecto que:

Se debe tener en cuenta que, en la práctica, más allá de que la medida de internamiento posea un carácter terapéutico, se lleva a cabo una auténtica privación de libertad de la persona. Por ello, tal como lo establecen los Principios de Salud Mental de las Naciones Unidas de 1991,el internamiento involuntario sólo debe tener un carácter excepcional y es necesario realizar todos los esfuerzos posibles para evitar el ingreso en contra de la voluntad del paciente" (Considerando 12). Así como también que, hoy nadie niega que las internaciones psiquiátricas que se prolongan innecesariamente son dañosas y conllevan, en muchos 
casos marginación, exclusión y maltrato y no es infrecuente que conduzcan a un "hospitalismo" evitable. (Considerando 6)

Por lo expuesto, debe recordarse, que la internación sólo podrá tomarse como medida de ultima ratio, ya que el Estado de conformidad con lo normado en el artículo 19 de la Convención sobre los Derechos de las Personas con Discapacidad y lo expresado por el Comité (2014) se encuentra obligado a propender al proceso de desinstitucionalización, garantizando que todas las personas con discapacidad recobren su capacidad jurídica y puedan elegir dónde y con quién vivir (42).

Finalmente, el Código prevé, en su artículo 47, que el cese de la incapacidad o de la restricción a la capacidad debe decretarse por el juez que la declaró, previo examen de un equipo interdisciplinario. Agregando que el juez puede ampliar la nómina de actos que la persona puede realizar por sí o con la asistencia de su curador o apoyo.

\section{II. c) Sistemas de apoyo al ejercicio de la capacidad}

El artículo 43 del Código Civil y Comercial de la Nación, en concordancia con lo previsto en el artículo 12 de la Convención sobre los Derechos de las Personas con Discapacidad define al apoyo como "cualquier medida de carácter judicial o extrajudicial que facilite a la persona que lo necesite la toma de decisiones para dirigir su persona, administrar sus bienes y celebrar actos jurídicos en general”. Asimismo, establece como función del mismo la de "promover la autonomía y facilitar la comunicación, la comprensión y la manifestación de voluntad de la persona para el ejercicio de sus derechos".

Se observa de este modo que el nuevo Código tiene como paradigma la autonomía de las personas con discapacidad, con lo cual toda intervención debe ser excepcional y tener como objeto la protección de sus intereses en pos del ejercicio pleno de sus derechos.

A su vez dicho cuerpo normativo dispone que el interesado puede proponer al juez la designación de personas de su confianza, debiendo el magistrado evaluar los alcances de la designación, la calidad de las medidas de apoyo, especificando las funciones con los ajustes razonables en función de las necesidades y circunstancias de la persona. Así como, debe procurar la protección de la misma respecto de eventuales conflictos de intereses o influencia indebida.

Considero pertinente, expresar que la persona con discapacidad o capacidad restringida debe contar con garantías de participación reales como son la accesibilidad, los ajustes razonables y apoyos durante el proceso de elección y propuesta referidos (Lorenzetti, 2014: 257). 
Caramelo, G.; Picasso, S. \& Herrera, M. (2015) señalan que pueden observase diferentes niveles de apoyos:

Un primer nivel es aquel en el que la persona requiere de apoyos mínimos para la toma de sus decisiones, como podrían serlo los relacionados con el lenguaje o con aspectos tecnológicos que puedan facilitar la comunicación. Un segundo nivel consiste en la toma de decisiones asistidas, en donde la persona con discapacidad recibe la asistencia para la toma de sus decisiones de un tercero de su confianza, elegido por la propia persona con discapacidad. Un tercer nivel es la toma de decisiones facilitada, para los casos extremos en que las preferencias y la voluntad no puedan expresarse o conocerse de manera fehaciente, y que debe constituirse en la situación de última instancia (115).

En el mismo orden de ideas, cabe recordar que el Comité de los Derechos de las Personas con Discapacidad (2014), señaló, que:

El tipo y la intensidad del apoyo que se ha de prestar variará notablemente de una persona a otra debido a la diversidad de las personas con discapacidad. Esto es acorde con lo dispuesto en el artículo $3 \mathrm{~d}$ ), en el que se describen como un principio general de la Convención: el respeto por la diferencia y la aceptación de las personas con discapacidad como parte de la diversidad y la condición humanas (16).

Por lo que, el apoyo deberá diseñarse según las necesidades de cada persona, debiendo respetarse siempre su autonomía individual y su capacidad para adoptar decisiones.

El apoyo podrá ser individual o colectivo, y conformarse por ejemplo por algún familiar o redes de familiares, por algún profesional o asociación, entre otros. Se proyecta tanto en los aspectos personales como en los patrimoniales (Lorenzetti, 2014: 251).

A su vez, cabe destacar, que a diferencia de la figura del curador, la actuación del apoyo no debe ser sustitutiva de la voluntad de las personas con discapacidad. Sino que tiene que propender a que la persona puede desarrollar su propio proceso de toma de decisiones, colaborando en caso de ser necesario con el proceso de razonamiento. Es decir, debe tener como eje la autonomía de la persona y el pleno ejercicio de sus derechos. Así como también "...fomentar la confianza y las aptitudes de las personas con discapacidad de modo que puedan ejercer su capacidad jurídica con menos apoyo en el futuro, si así lo desean” (CRDP, 2014: 20).

Teniendo la persona con capacidad restringida el derecho a "a rechazar el apoyo y poner fin a la relación de apoyo o cambiarla en cualquier momento" (CRDP, 2014: 24). 


\section{Conclusión}

El Código Civil y Comercial ha dado un paso importante en lo que se refiere a la adecuación de la legislación de fondo con la Convención sobre los Derechos de las Personas con Discapacidad.

En primer lugar, ha adaptado los términos a la Convención ya que utiliza vocablos tales como "apoyo", "ajustes razonables", "accesibilidad" y "autonomía".

Asimismo, ha pasado de un modelo tutelar de representación por sustitución - el cual se encontraba receptado en el código velezano - a un modelo basado en el sistema de apoyos. Es decir, recepta como una herramienta fundamental al "apoyo", garantizando de esta forma el ejercicio pleno de los derechos de las personas con discapacidad y respetando su autonomía en el proceso de la toma de decisiones.

Además, reafirma el criterio que establece la capacidad jurídica como regla, y prevé que sólo en casos excepcionales se podrá restringir la capacidad para determinados actos. Obligando al juez a revisar la sentencia cada vez que el interesado lo solicite y limitando las internaciones involuntarias.

A su vez, en reconocimiento de la autonomía de la persona con discapacidad y su derecho al acceso a la justicia, se le otorga un rol más activo a la misma durante el proceso de restricción de la capacidad.

Sin embargo, para que este avance legislativo no caiga en letra muerta, los actores involucrados - el juez, el equipo interdisciplinario, el Ministerio Público, los abogados, entre otros - deberán asumir el compromiso de garantizar a la persona con discapacidad su plena participación en el proceso y el respeto de su autonomía, para lograr así su plena inserción en la sociedad. 


\section{Bibliografía}

Borda, G. (1999). Tratado de Derecho Civil. Parte General. Tomo I. Buenos Aires: Abeledo Perrot. Recuperado de: http://www.cedesyc.com.ar/Material\%20de\%20estudio\%20UNR/Facultad\%20de\% 20derecho/Primer\%20a\%F1o/Tratado\%20de\%20Derecho\%20Civil\%20\%20Parte\%20General\%20-\%20Tomo\%201\%20Borda.pdf

Caramelo, G.; Picasso, S. \& Herrera, M. (coords.) (2015). Código civil y comercial de la Nación comentado. Tomo I ( $1^{\circ}$ Ed.) Buenos Aires: Infojus. Recuperado de http://www.saij.gob.ar/docs-f/codigocomentado/CCyC_Comentado_Tomo_I\%20(arts.\%201\%20a\%20400).pdf

Cifuentes, S. (1992). Elementos de Derecho Civil: Parte General. Buenos Aires: Astrea.

Código Civil y Comercial de la Nación [Código]. (2014). Recuperado de http://servicios.infoleg.gob.ar/infolegInternet/anexos/235000239999/235975/norm a.htm

Comité sobre los Derechos de las Personas con Discapacidad (2014). Observación general sobre el artículo 12: igual reconocimiento como persona ante la ley. Recuperado de http://webcache.googleusercontent.com/search?q=cache:i7dCJyVGod4J:www.ohchr.o $\mathrm{rg} /$ Documents/HRBodies/CRPD/GC/DGCArticle12_sp.doc $+\& \mathrm{~cd}=1 \&$ hl=es\&ct=cl $\mathrm{nk \& gl}=\mathrm{ar}$

Congreso de la Nación Argentina (6 de Junio de 2008) Convención sobre los Derechos de las Personas con Discapacidad y su Protocolo Facultativo. [Ley 26.378 de 2008]. Recuperado de http://servicios.infoleg.gob.ar/infolegInternet/anexos/140000144999/141317/norma.htm

Congreso de la Nación Argentina (2 de Diciembre de 2010). Ley Nacional de Salud Mental. [Ley 26.657 de 2010]. Recuperado de http://servicios.infoleg.gob.ar/infolegInternet/anexos/175000179999/175977/norma.htm

Congreso de la Nación Argentina (11 de Diciembre de 2014) Otórgase jerarquía constitucional a la Convención sobre los Derechos de las Personas con Discapacidad. [Ley 27.044 de 2014]. Recuperado de http://servicios.infoleg.gob.ar/infolegInternet/anexos/235000239999/239860/norma.htm

Corte Suprema de Justicia de la Nación. (19 de Febrero de 2008). Sentencia Competencia $\mathrm{N}^{\circ}$ 1195. XLII. R., M. J. s/ insania. Recuperado de http://www.saij.gob.ar/corte-suprema-justicia-nacion-federal-ciudad-autonoma-buenosaires-mj-insania-fa08000003-2008-02-19/123456789-300-0008-0ots-eupmocsollaf 
Fernández, S (2015). La capacidad jurídica de las personas con discapacidad en el nuevo Código Civil y Comercial bajo la lupa de los derechos humanos. Revista Código Civil y Comercial, vol 1 ( $\left.n^{\circ} 1\right)$.

Ferraiuolo, A \& Crocci, S. (2015). Capacidad jurídica y discapacidad. Breves referencias al sistema vigente. Derecho de Familia: Revista interdisciplinaria de doctrina y jurisprudencia, vol $1\left(n^{\circ} 2\right)$.

Lorenzetti, R (Director.) (2014). Código civil y comercial de la Nación comentado. Tomo I ( $1^{\circ}$ Ed.) Santa Fe: Rubinzal- Culzoni

Lorenzetti, R.; Higton de Nolasco, E. \& Kemelmajer de Carlucci, A. (2012) Proyecto de Código Civil y Comercial de la Nación. Buenos Aires: Infojus. Recuperado de http://www.saij.gob.ar/docsf/ediciones/libros/codigo_civil_comercial.pdf

Mackeldey, F. (1844). Elementos de Derecho Romano. Madrid: Imprenta de la Sociedad Literaria y Tipográfica.

Piccinelli, O. (2015) Ajustes razonables del proceso de declaración de incapacidad (Consideraciones a propósito del nuevo Código Civil y Comercial de la Nación). El Dial. Recuperado de https://www.eldial.com/nuevo/resultados-detalledoctrina.asp? $\mathrm{id}=8272 \&$ base $=50 \&$ resaltar $=$ piccinelli.

Toboso Martín, M. \& Arnau Ripollés, M. (2008). La discapacidad dentro del enfoque de capacidades y funcionamientos de Amartya Sen. Araucaria. Revista Iberoamericana de Filosofía, Política y Humanidades, vol 10 ( $n^{\circ}$ 20). Recuperado de https://www.fuhem.es/media/ecosocial/file/Boletin\%20ECOS/ECOS\%20CDV/Bo letin_8/Discapacidad_Amartya.pdf 